International Journal of Modern Physics E (C) World Scientific Publishing Company

\title{
Charming mesons with baryons and nuclei
}

\author{
Laura Tolos ${ }^{1,2} *$ \\ ${ }^{1}$ Instituto de Ciencias del Espacio (IEEC/CSIC), Campus Universitat Autònoma de Barcelona, \\ Facultat de Ciències, Torre C5, E-08193 Bellaterra (Barcelona), Spain \\ ${ }^{2}$ Frankfurt Institute for Advanced Studies, Johann Wolfgang Goethe University, \\ Ruth-Moufang-Str. 1, 60438 Frankfurt am Main, Germany
}

Received Day Month Year

Revised Day Month Year

\begin{abstract}
The properties of charmed mesons in nuclear matter and nuclei are reviewed. Different frameworks are discussed paying a special attention to unitarized coupled-channel approaches which incorporate heavy-quark spin symmetry. Several charmed baryon states with negative parity are generated dynamically by the $s$-wave interaction between pseudoscalar and vector meson multiplets with $1 / 2^{+}$and $3 / 2^{+}$baryons. These states are compared to experimental data. Moreover, the properties of open-charm mesons in matter are analyzed. The in-medium solution accounts for Pauli blocking effects, and for the meson self-energies in a self-consistent manner. The behavior in the nuclear medium of the rich spectrum of dynamically-generated baryon states is studied as well as their influence in the self-energy and, hence, the spectral function of open charm. The possible experimental signatures of the in-medium properties of open charm are finally addressed, such as the formation of charmed nuclei, in connection with the future FAIR facility.
\end{abstract}

Keywords: charmed mesons, unitarized coupled-channel theories, dynamically-generated states, nuclear matter and nuclei

PACS numbers: 14.20.Lq; 14.40.Lb; 21.65.Jk; 21.85.+d

\section{Introduction}

Quantum Chromodynamics (QCD) is considered to be the basic theory of the strong interaction. In the low-energy regime, QCD becomes a strongly-coupled theory, many aspects of which are not yet understood. A particular effort has been invested in exploring the QCD phase diagram for high density and/or temperatures. It is of great interest to understand the mechanisms inducing the transition from confined quarks inside hadrons to a deconfined plasma made of quarks and gluons as well as the restoration of certain QCD symmetries, such as the chiral symmetry in the light-quark sector, under extreme conditions of density and temperature Thus, the study of matter under extreme conditions has become one of the main research activities of several experimental programs, from the ongoing LHC/CERN

*e-mail: tolos@ice.csic.es 
project (Switzerland) 1 to the forthcoming PANDA and CBM experiments at FAIR (Germany) ${ }^{[2}$ Until now, due to the restrictions in the available energy, the studies have been concentrated in matter within the light-quark sector. With the on-going and upcoming research facilities, the aim is also to move from the light-quark domain to the heavy-quark one and to face new challenges where charm and new symmetries, such as heavy-quark symmetry, will play a dominant role.

Interest in the properties of open and hidden charmed mesons was triggered more than 20 years ago in the context of relativistic nucleus-nucleus collisions in connection with charmonium suppression ${ }^{3}$ as a probe for the formation of quarkgluon plasma (QGP). Nowadays, the nature of newly observed baryon and meson states with the charm degree of freedom is a matter of high interest in connection with many experiments, such as CLEO, Belle, BABAR ${ }^{4}[6]$ and others. The goal is to understand whether these states can be accommodated within the quark model picture and/or qualify better as being dynamically generated via hadronhadron scattering processes. To this end, a large part of the experimental program in hadronic physics at PANDA (FAIR) will be devoted to charmonium spectroscopy. Also, the CBM (FAIR) experiment will extend the GSI program for in-medium modification of hadrons in the light quark sector and provide the first insight into the charm-nucleus interaction. Indeed, the influence of medium modifications in the charmonium production at finite baryon densities would affect the formation of the QGP phase of QCD at high densities.

This review aims at overviewing the recent advances on the properties of charmed hadrons in dense matter and nuclei. We first start by analyzing baryon states with charm degrees of freedom in connection to experimental results. These states are generated dynamically within the context of unitarized meson-baryon coupled-channel models. We will pay a special attention to those which implement heavy-quark spin symmetry, which is a proper QCD symmetry in the limit of infinite quark masses. Then we implement medium corrections to the meson-baryon interaction in order to address the in-medium properties of open-charm mesons within self-consistent unitarized coupled-channel models. Finally, the formation of charmed mesic nuclei is considered as a possible experimental scenario to test charmed meson properties in matter at FAIR.

\section{Unitarized meson-baryon coupled-channel models with charm}

One of the primary goals in the physics of hadrons is to understand the nature of newly discovered states, whether they can be described within the quark model picture and/or as hadron-hadron molecules. In this section we will adopt the latter approach and analyze the recent developments in the description of experimental baryon states which incorporate the charm degree of freedom. These states are dynamically generated by means of the scattering of mesons and baryons in a unitarized coupled-channel description. It is, however, known that some baryon states can be constructed as $q q q$ entity in a quark model, and simultaneously as a 
dynamically generated state in a meson-baryon coupled-channel description (that is a $q q q-q \bar{q}$ molecular state) ${ }^{17}$ though, some of their properties might differ.

Given the success of unitarized coupled-channel approaches in the description of some of the existing experimental data in the light-quark sector, the charm degree of freedom has been recently incorporated in these models ${ }^{84}$ and several experimental states have been described as dynamically-generated baryon molecules. This is the case, for example, of the $\Lambda_{c}(2595)$, which is the charm sector counterpart of the $\Lambda(1405)$.

Whereas a separable potential for the bare meson-baryon interaction with only up, down and charm degrees of freedom was assumed in Ref., 8 some of these approaches are based on a bare meson-baryon interaction saturated with the $t$-channel exchange of vector mesons between pseudoscalar mesons and baryons in the zerorange approximation while preserving chiral symmetry for light mesons. ${ }^{10}[13] 15$ Later works revisited the zero-range approach by using the full $t$-dependence of the $t$-channel vector-exchange driving term. ${ }^{17}$ Other approaches have made use of the Jülich meson-exchange mode ${ }^{\sqrt{18} \mid 20}$ by deriving the transition potential in close analogy to the meson-exchange $\bar{K} N$ model while using, as a working hypothesis, $\mathrm{SU}(4)$ symmetry constraints. And some others have relied on the hidden gauge formalism 2124 to describe dynamically baryon states found experimentally.

All these models, however, do not explicitly incorporate heavy-quark spin symmetry (HQSS) 25 and, thus, it is unclear whether they fulfilled the constraints imposed by HQSS. a HQSS is a QCD symmetry that appears when the quark masses, such as the charm mass, become larger than the typical confinement scale. In the following, we analyze the implementation of HQSS in meson-baryon interactions as the charm degree of freedom is incorporated.

\subsection{Extended Weinberg-Tomozawa to spin-flavor with heavy-quark spin symmetry constraints}

HQSS predicts that all types of spin interactions involving heavy quarks vanish for infinitely massive quarks. Thus, HQSS connects vector and pseudoscalar mesons containing charmed quarks. On the other hand, chiral symmetry fixes the lowest order interaction between Goldstone bosons and other hadrons in a model independent way; this is the Weinberg-Tomozawa (WT) interaction. Thus, it is appealing to have a predictive model for four flavors including all basic hadrons (pseudoscalar and vector mesons, and $1 / 2^{+}$and $3 / 2^{+}$baryons) which reduces to the WT interaction in the sector where Goldstone bosons are involved and which incorporates HQSS in the sector where charm quarks participate. This model was developed in Refs. $\frac{29}{32}$ as an extension of the SU(6) approach in the light sector of Refs. $\frac{33}{38}$ Note that this model can be used not only for meson-baryon systems with charm

${ }^{a}$ A detailed analysis of the hidden-gauge models of Refs.21 2228 shows no actual violation in the heavy-quark limit (see Ref $[47$ ). 
content but also in the bottom sector, 39 since we expect HQSS to be better realized in heavier systems. Here we reproduce the main features.

We start with the extension of the WT meson-baryon interaction to spin-flavor symmetry (SF) with HQSS constraints. The extension for the on-shell vertex is ${ }^{33}$

$$
V_{\mathrm{WT}}^{\mathrm{sf}}=\frac{K(s)}{4 f^{2}} 4 J_{M}^{i} J_{B}^{i}, \quad i=1, \ldots,\left(2 N_{F}\right)^{2}-1,
$$

where $K(s)$ is a function that depends on the meson-baryon energy, and $J_{M}^{i}$ and $J_{B}^{i}$ are the $\mathrm{SU}\left(2 N_{F}\right)$ generators on mesons and baryons. Mesons consists of $0^{-}(P)$ and $1^{-}(V)$ lowest-lying states, while baryons contain $\frac{1}{2}^{+}(B)$ and $\frac{3}{2}^{+}\left(B^{*}\right)$ lowest-lying states. When this interaction is restricted to the sector $P B \rightarrow P B$, it reproduces the standard WT off $B$ targets.

The corresponding Hamiltonian for number of flavors $N_{F}$ and three colors reads 34

$$
\mathcal{H}_{\mathrm{WT}}^{\mathrm{sf}}(x)=-\frac{\mathrm{i}}{4 f^{2}}:\left[\Phi, \partial_{0} \Phi\right]^{A}{ }_{B} \mathcal{B}_{A C D}^{\dagger} \mathcal{B}^{B C D}:, \quad A, B, \ldots=1, \ldots, 2 N_{F},
$$

where $\Phi^{A}{ }_{B}(x)$ is the meson field, which contains the fields of $0^{-}$(pseudoscalar) and $1^{-}$(vector) mesons, and $\mathcal{B}^{A B C}$ is a completely symmetric tensor, which contains the fields of the lowest-lying baryons with $J^{P}=\frac{1}{2}^{+}$and $\frac{3}{2}^{+}$.

Extracting the kinematical part in Eq. (1), let

$$
H_{\mathrm{WT}}=4 J_{M}^{i} J_{B}^{i} .
$$

This operator can be written in terms of meson and baryon operators, 29,34 and it contains two distinct mechanisms which stem from expanding the meson commutator in Eq. (2),

$$
\begin{aligned}
H_{\mathrm{WT}} & =H_{\mathrm{ex}}+H_{\mathrm{ac}}, \\
H_{\mathrm{ex}} & =: M^{A}{ }_{C} M^{\dagger C}{ }_{B} B^{B D E} B_{A D E}^{\dagger}: \\
H_{\mathrm{ac}} & =-: M^{\dagger A}{ }_{C} M^{C}{ }_{B} B^{B D E} B_{A D E}^{\dagger}:, \quad A, \ldots, E=1, \ldots, 2 N_{F} .
\end{aligned}
$$

Here $M^{A}{ }_{B}$ and $B^{A B C}$ are the annihilation operators of mesons and baryons, respectively, with $M^{\dagger A}{ }_{B}=\left(M_{A}^{B}\right)^{\dagger}$, and $B_{A B C}^{\dagger}=\left(B^{A B C}\right)^{\dagger}$. $B^{A B C}$ is a completely symmetric tensor. They are normalized as

$$
\begin{aligned}
{\left[M_{B}^{A}, M^{\dagger C}{ }_{D}\right] } & =\delta_{D}^{A} \delta_{B}^{C}, \\
\left\{B^{A B C}, B_{A^{\prime} B^{\prime} C^{\prime}}^{\dagger}\right\} & =\delta_{A^{\prime}}^{A} \delta_{B^{\prime}}^{B} \delta_{C^{\prime}}^{C}+\cdots \quad(6 \text { permutations }) .
\end{aligned}
$$

Schematically, representing the quark and antiquark operators by $Q^{A}$ and $\bar{Q}_{A}$,

$$
M_{B}^{A} \sim Q^{A} \bar{Q}_{B}, \quad M_{B}^{\dagger A} \sim \bar{Q}^{\dagger A} Q_{B}^{\dagger}, \quad B^{A B C} \sim Q^{A} Q^{B} Q^{C}, \quad B_{A B C}^{\dagger} \sim Q_{A}^{\dagger} Q_{B}^{\dagger} Q_{C}^{\dagger} .
$$

So, an upper index in $M$ or $B$ represents the SF of a quark to be annihilated, whereas in $M^{\dagger}$ it represents that of an antiquark to be created. Likewise, a lower index in $M^{\dagger}$ or $B^{\dagger}$ represents the SF of a quark to be created while in $M$ it represents that of an antiquark to be annihilated. From this identification it is immediate 

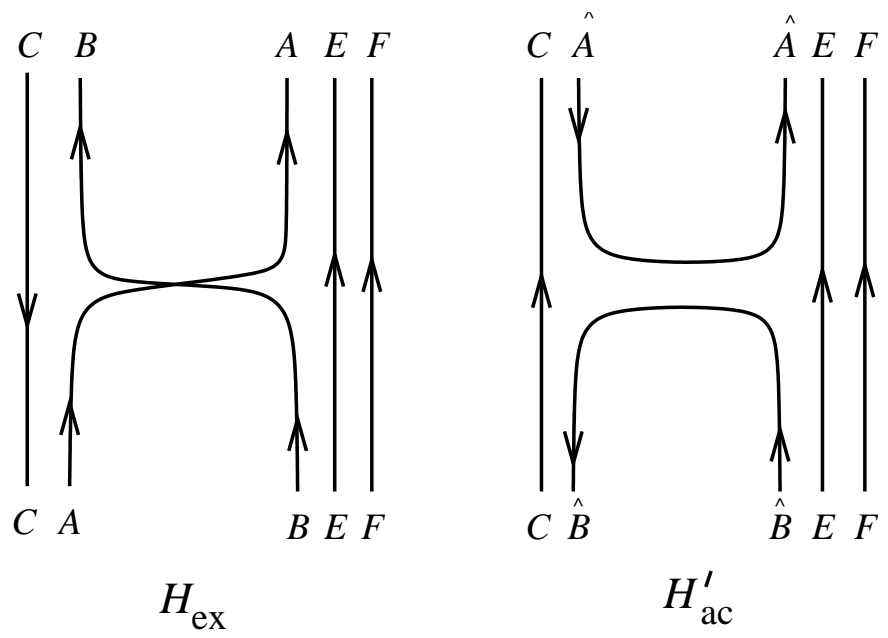

Fig. 1. The two mechanisms acting in the spin-flavor extended WT interaction. $H_{\mathrm{ex}}$ (exchange of quarks) and $H_{\mathrm{ac}}^{\prime}$ (annihilation and creation of quark-antiquark pairs) corrected by HQSS constraints. The indices $\hat{A}$ and $\hat{B}$ are restricted to light quarks.

to interpret the two mechanisms $H_{\mathrm{ex}}$ and $H_{\mathrm{ac}}$ in terms of quark and antiquark propagation: the exchange part $H_{\mathrm{ex}}$, in which a quark is transferred from the meson to the baryon, as another one is transferred from the baryon to the meson; and the annihilation-creation $H_{\mathrm{ac}}$ mechanism, where an antiquark in the meson annihilates with a similar quark in the baryon, with subsequent creation of a quark and an antiquark. The $H_{\mathrm{ac}}$ can violate HQSS when the annihilation or creation of $q \bar{q}$ pairs involve heavy quarks, as in the heavy-quark limit the number of charm quarks and the number of charm antiquarks are separately conserved (implying $\mathrm{U}_{c}(1) \times \mathrm{U}_{\bar{c}}(1)$ ). Indeed, the HQSS group, which also includes a group of separate rotations of the $c$ quark and $\bar{c}$ antiquark, reads as $\mathrm{HQSS}=\mathrm{SU}_{c}(2) \times \mathrm{SU}_{\bar{c}}(2) \times \mathrm{U}_{c}(1) \times \mathrm{U}_{\bar{c}}(1) . \mathrm{A}$ simple solution to enforce HQSS with minimal modifications is to remove just the offending contributions in $H_{\mathrm{ac}}$, which come from creation or annihilation of charm quark-antiquark pairs. This implies to remove the interaction when the labels $A$ or $B$ are of heavy type in $H_{\mathrm{ac}}$ to construct $H_{\mathrm{ac}}^{\prime}$ as

$$
H_{\mathrm{ac}}^{\prime}=-: M^{\dagger \hat{A}}{ }_{C} M^{C}{ }_{\hat{B}} B^{\hat{B} D E} B_{\hat{A} D E}^{\dagger}:, \quad C, D, E=1, \ldots, 8, \quad \hat{A}, \hat{B}=1, \ldots, 6 .
$$

The indices with hat are restricted to $\mathrm{SU}(6)$. The two final mechanisms that respect HQSS, $H_{\mathrm{ex}}$ and $H_{\mathrm{ac}}^{\prime}$ are shown in Fig. 1.

To summarize, the extended WT model to SF with HQSS constraints is given by

$$
V=\frac{K(s)}{4 f^{2}} H_{\mathrm{WT}}^{\prime}, \quad H_{\mathrm{WT}}^{\prime}=H_{\mathrm{ex}}+H_{\mathrm{ac}}^{\prime}
$$

This model fulfills some desirable requirements: i) it has symmetry $\mathrm{SU}(6) \times \mathrm{HQSS}$, 
i.e., SF symmetry in the light sector and HQSS in the heavy sector, the two invariances being compatible; ii) it reduces to $\mathrm{SU}(6)-\mathrm{WT}$ in the light sector, so it is consistent with chiral symmetry in that sector.

\subsection{Coupled-channel unitarization and symmetry breaking}

The final expression to be used for the extended WT interaction in the coupled meson-baryon basis for total charm $C$, strangeness $S$, isospin $I$ and spin $J$ is

$$
V_{i j}^{C S I J}=D_{i j}^{C S I J} \frac{2 \sqrt{s}-M_{i}-M_{j}}{4 f_{i} f_{j}} \sqrt{\frac{E_{i}+M_{i}}{2 M_{i}}} \sqrt{\frac{E_{j}+M_{j}}{2 M_{j}}},
$$

where $\sqrt{s}$ is the center of mass (C.M.) energy of the system; $E_{i}$ and $M_{i}$ are, respectively, the C.M. energy and mass of the baryon in the channel $i$; and $f_{i}$ is the decay constant of the meson in the $i$-channel. The hadron masses and meson decay constants can be found in Ref.31 b.

In order to calculate the scattering amplitudes, $T_{i j}$, we solve the on-shell BetheSalpeter equation (BSE), using the matrix $V^{C S I J}$ as kernel:

$$
T^{C S I J}=\left(1-V^{C S I J} G^{C S I J}\right)^{-1} V^{C S I J},
$$

where $G^{C S I J}$ is a diagonal matrix containing the meson-baryon propagator in each channel. Explicitly,

$$
G_{i i}^{C S I J}=2 M_{i}\left(\bar{J}_{0}\left(\sqrt{s} ; M_{i}, m_{i}\right)-\bar{J}_{0}\left(\mu^{S I} ; M_{i}, m_{i}\right)\right),
$$

with $M_{i}\left(m_{i}\right)$ the mass of the baryon (meson) in the channel $i$. The loop function $\bar{J}_{0}$ can be found in the appendix of Ref. ${ }^{40}$ (Eq. A9) for the different possible Riemann sheets. The meson-baryon propagator (loop) is logarithmically ultraviolet divergent, thus, it needs to be renormalized. This has been done by a subtraction point regularization such that

$$
G_{i i}^{C S I J}(\sqrt{s})=0 \text { at } \sqrt{s}=\mu^{C S I},
$$

with $\mu^{C S I}=\sqrt{m_{\mathrm{th}}^{2}+M_{\mathrm{th}}^{2}}$, where $m_{\mathrm{th}}$ and $M_{\mathrm{th}}$, are, respectively, the masses of the meson and baryon producing the lowest threshold (minimal value of $m_{\mathrm{th}}+M_{\mathrm{th}}$ ) for each CSI sector, independent of the angular momentum $J$. This renormalization scheme was first proposed in Refs. ${ }^{12}[13$ and it was successfully used in Refs 29,3139 A 39 recent discussion on the regularization method can be found in Ref. 42

The dynamically-generated baryon states appear as poles of the scattering amplitudes on the complex energy $\sqrt{s}$ plane. The poles of the scattering amplitude

\footnotetext{
${ }^{\mathrm{b}}$ Other alternative expression for the WT can be found in Ref ${ }^{[32}$ In this work, the $\left(2 \sqrt{s}-M_{i}-M_{j}\right)$ term, with $M_{i}$ and $M_{j}$ the incoming and outgoing baryon masses, has been approximated by the sum of the CM energies of the incoming and outgoing mesons. This non-relativistic approximation for the baryons is consistent with the treatment for the baryons to implement the HQSS constraints. Moreover, the $\sqrt{(E+M) /(2 M)}$ factors included in the potential have been moved to the definition of the loop function.
} 
on the first Riemann sheet that appear on the real axis below threshold are interpreted as bound states. The poles that are found on the second Riemann sheet below the real axis and above threshold are identified with resonances. The mass and the width of the state can be found from the position of the pole on the complex energy plane. Close to the pole, the scattering amplitude behaves as

$$
T_{i j}^{C S I J}(s) \approx \frac{g_{i} e^{i \phi_{i}} g_{j} e^{i \phi_{j}}}{\sqrt{s}-\sqrt{s_{R}}} .
$$

The mass $M_{R}$ and width $\Gamma_{R}$ of the state result from $\sqrt{s_{R}}=M_{R}-\mathrm{i} \Gamma_{\mathrm{R}} / 2$, while $g_{j} e^{i \phi_{j}}$ (modulus and phase) is the coupling of the state to the $j$-channel.

The dynamically-generated states can be classified under the symmetry group $\mathrm{SU}(6) \times$ HQSS. Indeed, dynamically generated states are most likely to occur coming from the most attractive $\mathrm{SU}(6) \times$ HQSS representations. However, the $\mathrm{SU}(6) \times$ HQSS is strongly broken in nature and several soft symmetry-breaking mechanisms have to be introduced in order to compare the dynamically-generated states to the experimentally observed ones. This is performed by an adiabatic change of hadron masses and meson weak-decay constants. We consider the breaking of the light SF $\mathrm{SU}(6)$ to $\mathrm{SU}(3) \times \mathrm{SU}_{J_{l}}(2)$, with $J_{l}$ the spin of the light quarks. Subsequently, we break the $\mathrm{SU}(3)$ light flavor group to $\mathrm{SU}(2)$ isospin symmetry group, preserving the HQSS. Thus, we assume exact isospin, total spin and flavor conservation. In this way we can assign $\mathrm{SU}(3)$ and $\mathrm{SU}(6)$ representation labels to each found state, and also identify the HQSS multiplets.

\section{Dynamically-generated baryon states with charm content}

The experimental status on charmed baryon states is summarized in Table 1. Most of the states have been observed in the $C=1$ sector with zero strangeness. Some of these states can be identified with dynamically generated resonances $\mathrm{C}$ using unitarized meson-baryon coupled-channel models. In the following we present the results using the extended WT interaction to SF with HQSS constraints as well as results from other unitarized coupled-channel schemes. This identification is made by comparing the PDG data ${ }^{43}$ on these states with the mass, width and, most important, the coupling to the meson-baryon channels of our dynamically-generated poles.

\section{1. $\mathrm{C}=1,2,3$ baryon states}

We start by showing the results for the $C=1,2,3$ sectors, where the meson-baryon channels with $c \bar{c}$ pairs have been dropped out. We will consider in the last subsection the case when a $c \bar{c}$ is present, but only for the $C=0$ case.

${ }^{\mathrm{c}}$ Often we refer to all poles generically as resonances, regardless of their concrete nature, since usually they can decay through other channels not included in the model space. 


\begin{tabular}{lccccc}
\hline Resonance & $I\left(J^{P}\right)$ & Status & Mass $[\mathrm{MeV}]$ & $\Gamma[\mathrm{MeV}]$ & $\begin{array}{c}\text { Prediction } \\
\text { Mass }[\mathrm{MeV}](\Gamma[\mathrm{MeV}])\end{array}$ \\
\hline$\Lambda_{c}(2595)$ & $0\left(1 / 2^{-}\right)$ & $* * *$ & $2592.25 \pm 0.28$ & $2.6 \pm 0.6$ & $2618.8(1.2)$ \\
$\Lambda_{c}(2625)$ & $0\left(?^{?}\right)$ & $* * *$ & $2628.11 \pm 0.19$ & $<0.97$ & $2666.6(53.7)$ \\
$\Lambda_{c}(2765)$ & $?\left(?^{?}\right)$ & $*$ & $2766.6 \pm 2.4$ & 50 & \\
or $\Sigma_{c}(2765)$ & & & & & \\
$\Lambda_{c}(2880)$ & $0\left(5 / 2^{+}\right)$ & $* * *$ & $2881.53 \pm 0.35$ & $5.8 \pm 1.1$ & \\
$\Lambda_{c}(2940)$ & $0\left(?^{?}\right)$ & $* * *$ & $2939.8_{-1.5}^{+1.4}$ & $17_{-6}^{+8}$ & \\
$\Sigma_{c}(2800)^{++}$ & $1\left(?^{?}\right)$ & $* * *$ & $2801_{-6}^{+4}$ & $75_{-17}^{+22}$ & \\
$\Sigma_{c}(2800)^{+}$ & $1\left(?^{?}\right)$ & $* * *$ & $2792_{-5}^{+14}$ & $62_{-40}^{+60}$ & \\
$\Sigma_{c}(2800)^{0}$ & $1\left(?^{?}\right)$ & $* * *$ & $2806_{-7}^{+5}$ & $72_{-15}^{+22}$ & \\
$\Xi_{c}(2790)^{+}$ & $1 / 2\left(?^{?}\right)$ & $* * *$ & $2789.1 \pm 3.2$ & $<15$ & $2804.8(20.7)$ \\
$\Xi_{c}(2790)^{0}$ & $1 / 2\left(?^{?}\right)$ & $* * *$ & $2791.8 \pm 3.3$ & $<12$ & \\
$\Xi_{c}(2815)^{+}$ & $1 / 2\left(?^{?}\right)$ & $* * *$ & $2816.6 \pm 0.9$ & $<3.5$ & $2845.2(44.0)$ \\
$\Xi_{c}(2815)^{0}$ & $1 / 2\left(?^{?}\right)$ & $* * *$ & $2819.6 \pm 1.2$ & $<6.5$ & \\
$\Xi_{c}(2930)$ & $1 / 2\left(?^{?}\right)$ & $*$ & $2931 \pm 6$ & $36 \pm 13$ & \\
$\Xi_{c}(2980)^{+}$ & $1 / 2\left(?^{?}\right)$ & $* * *$ & $2971.4 \pm 3.3$ & $26 \pm 7$ & \\
$\Xi_{c}(2980)^{0}$ & $1 / 2\left(?^{?}\right)$ & $* * *$ & $2968.0 \pm 2.6$ & $20 \pm 7$ & \\
$\Xi_{c}(3055)$ & $1 / 2\left(?^{?}\right)$ & $* *$ & $3054.2 \pm 1.3$ & $17 \pm 13$ & \\
$\Xi_{c}(3080)^{+}$ & $1 / 2\left(?^{?}\right)$ & $* * *$ & $3077.0 \pm 0.4$ & $5.8 \pm 1.0$ & \\
$\Xi_{c}(3080)^{0}$ & $1 / 2\left(?^{?}\right)$ & $* * *$ & $3079.9 \pm 1.4$ & $5.6 \pm 2.2$ & \\
$\Xi_{c}(3123)$ & $1 / 2\left(?^{?}\right)$ & $*$ & $3122.9 \pm 1.3$ & $4 \pm 4$ & \\
\hline
\end{tabular}

Table 1. Summary of experimental data for baryon resonances in the different charm sectors as compiled in Ref. ${ }^{43}$ The lowest-lying $\Lambda_{c}, \Sigma_{c}, \Sigma_{c}^{*}, \Xi_{c}, \Xi_{c}^{\prime}, \Xi_{c}^{*}, \Omega_{c}, \Omega_{c}^{*}, \Xi_{c c}, \Xi_{c c}^{*}, \Omega_{c c}, \Omega_{c c}^{*}$ and $\Omega_{c c c}$ states are omitted. The mass and the width of the dynamically-generated states using the extended WT to SF with HQSS constraints are shown in the last column. The predicted values for $\Xi_{c}(2790)$ and $\Xi_{c}(2815)$ are the isospin-average states.

In the $C=1, S=0, I=0, J=1 / 2$ case, we obtain three states in the $\Lambda_{c}$ sector, which come from the most attractive $\mathrm{SU}(6) \times \mathrm{HQSS}$ representations (irrep), denoted by $\mathbf{1 5}_{\mathbf{2}, \mathbf{1}}$ and $\mathbf{2 1}_{\mathbf{2}, \mathbf{1}}$. Here we use the notation $R_{2 J_{C}+1, C}$, where $\mathbf{R}$ is the $\mathrm{SU}(6)$ irrep label (for which we use the dimension) and $J_{C}$ is the spin carried by the quarks with charm. The experimental $\Lambda_{c}(2595)$ resonance can be identified with the pole that we obtain around $2618.8 \mathrm{MeV}$. The width is, however, smaller than the experimental result since the dominant three-body decay channel $\Lambda_{c} \pi \pi^{43}$ is not included in our calculation. A second broad $\Lambda_{c}$ resonance at $2617.3 \mathrm{MeV}$ is observed with a large coupling to the open channel $\Sigma_{c} \pi$, very close to $\Lambda_{c}(2595)$. This is the same two-pole pattern found in the charmless $I=0, S=-1$ sector for the $\Lambda(1405){ }^{44}$ A third spin- $1 / 2 \Lambda_{c}$ resonance is seen around $2828 \mathrm{MeV}$ and cannot be assigned to any experimentally known resonance. With regard to spin-3/2 resonances, we find one located at $(2666.6-i 26.8 \mathrm{MeV})$ coming from the attractive $\mathbf{1 5}_{\mathbf{2}, \mathbf{1}}$ irrep. This resonance is assigned to the experimental $\Lambda_{c}(2625)$ and forms 
with the broad 2617.3 resonance a HQSS doublet. The $t$-channel vector-exchange model of Ref $\frac{13}{13}$ also reported a similar resonance at $2660 \mathrm{MeV}$. The novelty of our calculations with respect to that one is that we obtain a non-negligible contribution from the vector meson-baryon channels to the generation of this resonance.

For $C=1, S=0, I=1, J=1 / 2$ ( $\Sigma_{c}$ sector $)$, three $\Sigma_{c}$ resonances are obtained from $\mathbf{1 5}_{\mathbf{2}, \mathbf{1}}$ and $\mathbf{2 1}_{\mathbf{2}, \mathbf{1}}$ irreps with masses $2571.5,2622.7$ and $2643.4 \mathrm{MeV}$ and widths 0.8, 188.0 and $87.0 \mathrm{MeV}$, respectively. Those states are pure predictions of our model. Ref. ${ }^{[17}$ predicts the existence of two resonances in this sector. However, only one of them can be identified to one of ours but with a strong vector meson-baryon component. Moreover, we predict two spin- $3 / 2 \Sigma_{c}$ resonances appearing from $\mathbf{2 1}_{\mathbf{2}, \mathbf{1}}$ irrep. The first one, a bound state at $2568.4 \mathrm{MeV}$, is thought to be the charmed counterpart of the $\Sigma(1670)$. The second state at $2692.9-i 33.5 \mathrm{MeV}$ has not a direct experimental comparison.

Our model generates six $\Xi_{c}$ states with $J=1 / 2$ and three with $J=3 / 2$ from

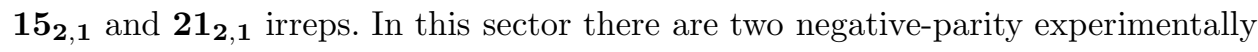
known resonances that can be identified with some of our dynamically-generated states, namely experimental $\Xi_{c}(2790) J^{P}=1 / 2^{-}$and $\Xi_{c}(2815) J^{P}=3 / 2^{-}$. The state $\Xi_{c}(2790)$ has a width of $\Gamma<12-15 \mathrm{MeV}$ and it decays to $\Xi_{c}^{\prime} \pi$, with $\Xi_{c}^{\prime} \rightarrow \Xi_{c} \gamma$. We assign it to the $2804.8-i 10.3 \mathrm{MeV}$ state found in our model because of the large $\Xi_{c}^{\prime} \pi$ coupling. A slight modification of the subtraction point can lower the position of our resonance to $2790 \mathrm{MeV}$ and most probably reduce its width as it will get closer to the $\Xi_{c}^{\prime} \pi$ channel. It could be also possible to identify our pole at $2733 \mathrm{MeV}$ with the experimental $\Xi_{c}(2790)$ state. In that case, one would expect that if the resonance position gets closer to the physical mass of $2790 \mathrm{MeV}$, its width will increase and it will easily reach values of the order of $10 \mathrm{MeV}$. With regards to the $\Xi_{c}$ resonance with $J^{P}=3 / 2^{-}$, the full width of the experimental $\Xi_{c}$ is expected to be less than $3.5 \mathrm{MeV}$ for $\Xi_{c}^{+}(2815)$ and less than $6.5 \mathrm{MeV}$ for $\Xi_{c}^{0}(2815)$, and the decay modes are $\Xi_{c}^{+} \pi^{+} \pi^{-}, \Xi_{c}^{0} \pi^{+} \pi^{-}$. We obtain two resonances at $2819.7-i 16.2 \mathrm{MeV}$ and $2845.2-i 22.0 \mathrm{MeV}$, respectively, that couple strongly to $\Xi_{c}^{*} \pi$, with $\Xi_{c}^{*} \rightarrow \Xi_{c} \pi$. Allowing for this possible indirect three-body decay channel, we might identify one of them to the experimental result. This assignment is possible for the state at $2845.2 \mathrm{MeV}$ if we slightly change the subtraction point, which will lower its position and reduce its width as it gets closer to the threshold of the open $\Xi_{c}^{*} \pi$ channel. With this assignment, we find that the experimental $\Xi_{c}(2790)$ and $\Xi_{c}(2815)$ are HQSS partners.

Three $\Omega_{c}$ bound states are obtained from $\mathbf{1 5}_{\mathbf{2}, \mathbf{1}}$ and $\mathbf{2 1}_{\mathbf{2 , 1}}$ irreps with masses $2810.9,2884.5$ and $2941.6 \mathrm{MeV}$. There is no experimental information on those excited states. However, our predictions can be compared to recent calculations of Refs ${ }^{12}\left[17\right.$ In Ref ${ }^{[17}$ three $\Omega_{c}$ resonances are predicted, with masses higher than ours by approximately $100 \mathrm{MeV}$. Further, we obtain two spin-3/2 bound states $\Omega_{c}$ from the attractive $\mathbf{2 1}_{\mathbf{2}, \mathbf{1}}$ irrep with masses 2814.3 and $2980.0 \mathrm{MeV}$, which mainly couple to $\Xi D^{*}$ and $\Xi^{*} D^{*}$, and to $\Xi_{c}^{*} \bar{K}$, respectively. As in the $J=1 / 2$ sector, no experimental information is available here. 
No experimental information is available in $C=2\left(\Xi_{c} c\right.$ and $\left.\Omega_{c c}\right)$ and $C=3$ $\left(\Omega_{c c c}\right)$. Several states are generated dynamically in our model coming from the $\mathrm{SU}(6) \times \operatorname{HQSS} \mathbf{6}_{\mathbf{1 , 2}}$ and $\mathbf{6}_{\mathbf{3}, \mathbf{2}}$ representations $\left(\Xi_{c c}\right.$ and $\Omega_{c c}$ states), while from $\mathbf{1}_{\mathbf{2}, \mathbf{3}}$ and $\mathbf{1}_{\mathbf{4}, \mathbf{3}}$ for $\Omega_{c c c}$, all of them bound states in the $\Omega_{c c}$ and $\Omega_{c c c}$ sectors. For $\Omega_{c c c}$ the large separation from the closest threshold suggests that interaction mechanisms beyond $s$-wave could be relevant for the formation of the dynamically-generated states with $C=3$.

\section{2. $C=-1$ baryon states}

A very rich spectrum of exotic states is obtained in the $C=-1$ sector. In this case, only meson-baryon states with one $\bar{c}$ quark are considered. For $J=1 / 2$ we find $6 \mathrm{SU}(3)$ multiplets of resonances, while for $J=3 / 2$ our model generates 5 $\mathrm{SU}(3)$ multiplets and a $\mathrm{SU}(3)$ multiplet in $J=5 / 2$. The predictions of Refs. 12 [13 within a $t$-channel vector exchange model and in Ref ${ }^{45}$ in the Skyrme model are reproduced with the extended WT interaction. However, our states turn out to be about $100-200 \mathrm{MeV}$ heavier. Using the extended WT interaction new states are also predicted because of the inclusion of vector mesons.

Among the resonances we call the attention to a $C=-1, S=0, I=0, J=1 / 2$ state generated by the $\bar{D} N$ and $\bar{D}^{*} N$ coupled channel dynamics. This state appears bound by only $1 \mathrm{MeV}$, and it is one of our more interesting predictions. Moreover, it appears as a consequence of treating heavy pseudoscalars and heavy vector mesons on an equal footing, as required by HQSS because no resonance would be generated unless $\bar{D}^{*} N$ channel is considered.

One of the poles we obtain for $J=3 / 2$ has a mass close to $3100 \mathrm{MeV}$. There is one experimental claim for an exotic state with $C=-1$ around this mass in Ref. $\frac{46}{6}$ The state has been observed in the decay mode

$$
\Theta_{\bar{C}} \rightarrow \bar{D}^{*} N \rightarrow \bar{D} \pi N
$$

Our dynamically generated state has other two possible decay channels induced by its coupling to channels involving the $\Delta(1232)$ resonance, which is not a stable particle. Thus, the anti-charmed resonance can decay to $\bar{D}$ or $\bar{D}^{*}$ plus a virtual $\Delta$, which subsequently would decay into a $\pi N$ pair. So, the dynamically generated state has another two decay mechanisms apart from the one in Eq. (14), namely,

$$
\begin{aligned}
& \Theta_{\bar{C}} \rightarrow \bar{D} \Delta \rightarrow \bar{D} \pi N \\
& \Theta_{\bar{C}} \rightarrow \bar{D}^{*} \Delta \rightarrow \pi \bar{D} \pi N .
\end{aligned}
$$

The decay in Eq. (16) has the same particles in the final state that in Eq. (14), with the difference that the pion in one case is coming from the decay of the $\bar{D}^{*}$ and therefore has low momentum, while in the other channel it comes from a $\Delta$ and may have higher momentum. The experimental search made in Ref. ${ }^{46}$ looked only for pions in order to reconstruct a $\bar{D}^{*}$ and may have missed the other events where the pion comes from a $\Delta$. 


\subsection{Hidden-charm baryon states: the $C=0$ case}

In this section we analyze the hidden charm sector, that is, the dynamically generated states with $c \bar{c}$ pairs stemming from meson-baryon interactions. We focus on the $C=0$ case, so that the resonances can be labeled as $N$ - and $\Delta$-states. These states stem from the two attractive $\mathrm{SU}(6) \times$ HQSS representations in the hidden-charm sector with $C=0$, i.e., $\mathbf{5 6}_{\mathbf{2}, \mathbf{0}}$ and $\mathbf{7 0}_{\mathbf{2}, \mathbf{0}}$ irreps. Due to the presence of a $c \bar{c}$ pair, these $N$ and $\Delta$ states have masses around $4 \mathrm{GeV}$ and no experimental counterparts for these states have been found yet.

We find three $N_{1 / 2}$ (the lower index indicates $J$ ), three $N_{3 / 2}$, and one $N_{5 / 2}$, with masses between 3918 and $4027 \mathrm{MeV}$. Some of the states are degenerated when the HQSS is unbroken, thus forming the HQSS multiplets. Almost all found $N$ resonances are bound states.

Compared to other works, the $N$ resonances studied in the zero-range $t$-channel vector-meson exchange model of Refs ${ }^{12 \mid 13}$ are about $500 \mathrm{MeV}$ lighter than those found in our model. The hidden-gauge formalism predicts these masses to be about $400 \mathrm{MeV}$ larger!22]23]47] However, this difference comes mostly from using a different renormalization prescription. ${ }^{47}$ Our results are close to those predicted by the chiral interaction, studied in the constituent quark model of Ref., ${ }^{48}$ whereas the instantoninduced interaction and color-magnetic interaction produce higher masses for the resonances $\underline{48}$

Further, we find three $\Delta_{1 / 2}$ and two $\Delta_{3 / 2}$ bound states. Two of them, $\Delta_{1 / 2}(4306)$ and $\Delta_{3 / 2}(4307)$, which stem from the $\mathbf{5 6}_{\mathbf{2}, \mathbf{0}}$ representation, appear as cusps in the scattering amplitude.

\section{Open charm in dense nuclear matter}

The mass and width of the dynamically-generated states described in the previous section can be modified once these states are obtained in a dense medium. This is a direct consequence of the changes in matter experienced by the mesons and baryons that generate these states. The properties of open-charm mesons in matter have been object of a recent theoretical interest due to the consequences for charmonium suppression, as observed at SPS energies by the NA50 collaboration. ${ }^{49}$ The change of the properties of D mesons in matter should modify the $J / \Psi$ absorption in a hot

and dense nuclear medium and can provide an explanation for $J / \Psi$ suppression $50+53$ Furthermore, there have been speculations about the existence of $D$ meson bound states in nucle ${ }^{54}$ resulting from a strong attractive potential felt by the $D$ mesons in nuclear matter. 55

Several theoretical works have addressed the properties of open-charm mesons in matter. While some studies have been devoted to the properties of open charm with light mesons,,$\sqrt[567]{\mid 57}$ over the past decade the interest has been also focused on open charm at finite baryonic density. A phenomenological estimate based on the quark-meson coupling (QMC) model,$\frac{58}{5}$ which is based on the exchange of $\omega, \rho$ and $\sigma$ mesons among the quarks in the meson/baryon bag, predicts an attractive 
$D^{+}$-nucleus potential at normal nuclear matter density $\left(\rho_{0}\right)$ of the order of -140 $\mathrm{MeV} ! 55$ The $D$-meson mass shift has also been studied using the QCD sum-rule (QSR) approach ${ }^{59}[63$ In these works the mass modification of the $D$-meson has a large contribution from the light quark condensates. A mass shift of $-50 \mathrm{MeV}$ at $\rho_{0}$ has been suggested ${ }^{[59}$ A second analysis based on QSR, however, predicts only a splitting of $D^{+}$and $D^{-}$masses of $60 \mathrm{MeV}$ at $\rho_{0}$ because the uncertainties to which the mass shift is subject at the level of the unknown $D N$ coupling to the sector of charm baryons and pions 60 Recent results on QSR rules for open-charm mesons can be found in ${ }^{61] 63}$ On the other hand, the mass modification of the $D$-meson has been also addressed using a chiral effective model in hot and dense matter $\frac{64}{66}$ within the mean field or relativistic Hartree Fock approaches, and strong mass shifts were obtained. Whereas similar results to the previous works on QMC model or QSR are obtained at $T=0$ with the interaction Lagrangian of chiral perturbation theory, a larger mass drop of $\sim-200 \mathrm{MeV}$ at $T=0$ is observed when a $\mathrm{SU}(4)$ effective model is use. At finite temperature, the attraction is, though, reduced.

Nevertheless, in all these investigations the full spectral features (mass and width) of the open-charm mesons in dense nuclear matter have not been considered. Thus, a self-consistent unitarized coupled-channel approach is required.

\subsection{Self-consistent unitarized meson-baryon coupled-channel schemes}

The spectral features of open-charm mesons and, hence, the self-energy in nuclear matter are obtained by incorporating the corresponding medium modifications in the effective open charm-nucleon interactions. This amounts for solving a unitarized coupled-channel equation similar to Eq. (10), where the intermediate mesonbaryon propagators contain different sources of density dependence. This procedure requires the implementation of self-consistency for open charm. Indeed, the effective open charm-nucleon interactions in matter are used to calculate the open-charm spectral features (masses and widths). These features modify the intermediate open charm-nucleon propagators which enter in the calculation of the effective interactions in matter and, thus, the solution requires a self-consistent treatment.

The $D$ meson mass and width were first obtained in the exploratory work of Ref $[8$ within a self-consistent coupled-channel approach assuming a separable potential for the bare meson-baryon interaction. Finite temperature effects in the $D$ mesonnucleon interaction were incorporated later on in Ref. ${ }^{[9}$ The spectral function for $D$ mesons can be seen in the left-hand plot of Fig. 2. Within the $t$-channel vectormeson exchange model of Refs., $12\left[13\right.$ the spectral features of the $D, \bar{D}$ and $D_{s}, \bar{D}_{s}$ mesons in nuclear matter were analyzed in the zero-range limit 14 These results were revisited in Refs. $\frac{15}{16}$ The results are shown in the right-hand plot of Fig. 2 for the first model while in the left-hand plot of Fig. 3 for the revisited one. More recently, the spectral features of $D, \bar{D}$ and $D_{s}, \bar{D}_{s}$ mesons have been addressed taking for the interaction kernel a $t$-channel vector meson exchange model beyond the zero-range 

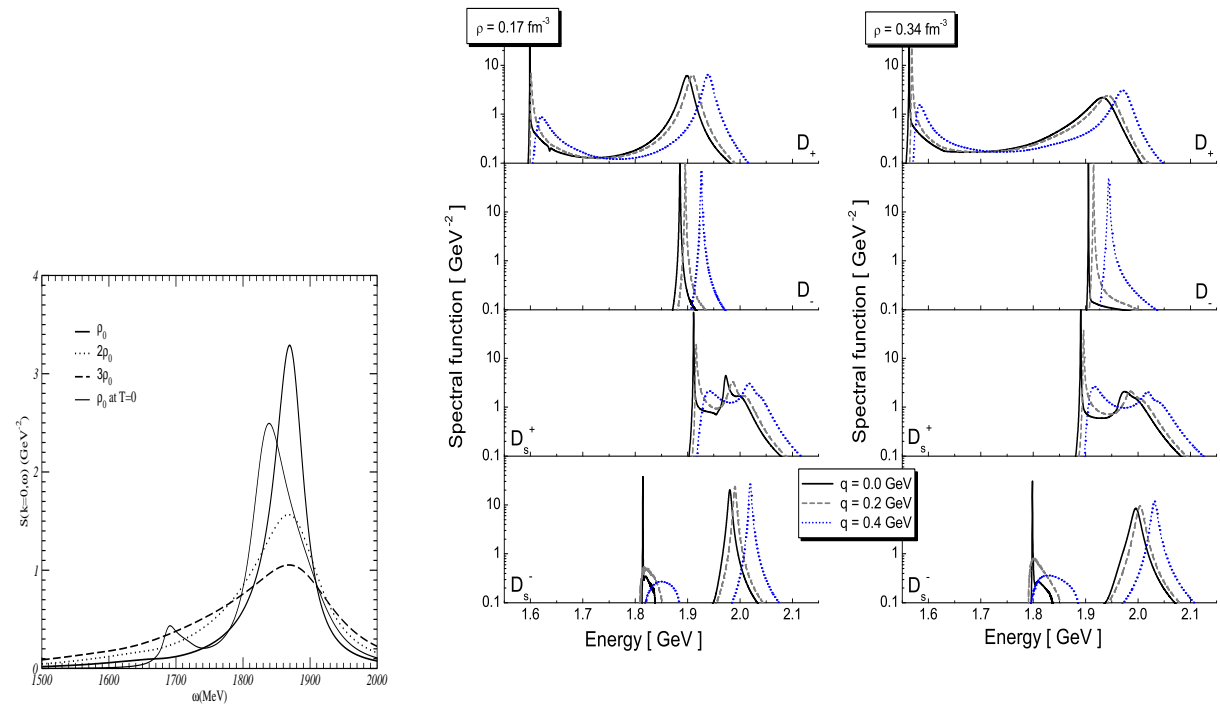

Fig. 2. Left figure (taken from Ref ${ }^{9}$ ): The $D$ spectral function at zero momentum for different densities at $T=120 \mathrm{MeV}$ together with the $\rho_{0}, T=0$ case within the self-consistent coupledchannel approach that assumes a separable potential for the bare meson-baryon interaction of Ref $[9]$. Right figure (taken from Ref ${ }^{14}$ ): The $D, \bar{D}, D_{s}$ and $\bar{D}_{s}$ spectral functions at different momenta for $\rho_{0}$ and $2 \rho_{0}$ within the $t$-channel vector-meson exchange model in matter of Ref $[14$.

approximation $\frac{67}{6}$ (see right-hand plot of Fig. 3 for the $D$ meson spectral function).

These models in dense matter, however, are not consistent with HQSS, as heavy vector mesons and pseudoscalars are not treated on equal footing. In the following, we address the implementation of HQSS constraints in the determination of the spectral features of open-charm mesons.

\subsection{Implementation of heavy-quark spin symmetry}

As seen in Sec. 2.1. a spin-flavor symmetric model for four flavors that implements HQSS constraints has been developed in Refs $29 \sqrt{32} 39$, similarly to the SU(6) approach in the light sector of Refs. ${ }^{33}\left[38\right.$. This model implements HQSS ${ }^{25}$ and dynamically generates resonances with negative parity in all the isospin, spin, strange and charm sectors that one can form from an s-wave interaction between pseudoscalar and vector meson multiplets with $1 / 2^{+}$and $3 / 2^{+}$baryons.

Within this model, the properties of open-charm mesons in nuclear matter can be obtained by incorporating the corresponding medium modifications in the effective open charm-nucleon interactions. One of the sources of density dependence comes from the Pauli principle acting on the nucleons, which prevents the scattering to already occupied nucleon states. Another source is related to the change of the properties of mesons and baryons in the intermediate states of the coupled-channel 

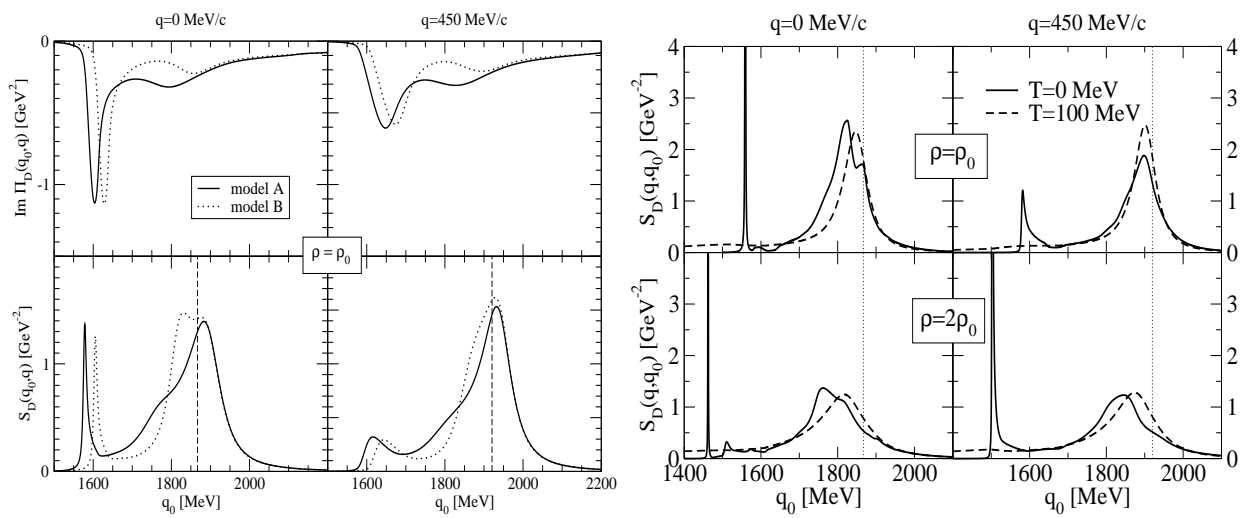

Fig. 3. Left figure (taken from Ref ${ }^{15}$ ): The $D$ meson self-energy (upper panels) and spectral function (lower panels) at $\rho=\rho_{0}$ for two momenta within the $t$-channel vector-meson exchange model in the zero-range approximation of Ref 15 . Two different approaches are depicted. Right figure (taken from Ref $\left[67\right.$ ): The $D$ meson spectral function for $\rho_{0}$ and $2 \rho_{0}$, for different momenta and temperatures within the model that uses a $t$-channel vector meson exchange approach beyond the zero-range approximation of Ref $[67$.

structure due to the interaction with nucleons of the Fermi sea. Those changes are implemented by using the in-medium meson-baryon propagators instead of the corresponding free ones. as 68

The renormalized (finite) loop function in free space of Eq. (11) can be rewritten

$$
G^{0}(\sqrt{s})=\mathrm{i} 2 M \int \frac{d^{4} q}{(2 \pi)^{4}}\left(D_{\mathcal{B}}^{0}(P-q) D_{\mathcal{M}}^{0}(q)-D_{\mathcal{B}}^{0}(\bar{P}-q) D_{\mathcal{M}}^{0}(q)\right)
$$

where $D_{\mathcal{B}(\mathcal{M})}^{0}$ is the free baryon (meson) propagator and with $P$ and $\bar{P}$ defined such that $P^{2}=s, \bar{P}^{2}=(\mu)^{2}$, with $\mu$ the subtraction point. For simplicity, the $C, S, I$ and $J$ indices have been omitted.

In matter, the medium changes appear as a correction to the renormalized (finite) loop function of free space ${ }^{68}$

$$
\begin{aligned}
G_{\rho}(P) & =G^{0}(\sqrt{s})+\delta G_{\rho}(P), \\
\delta G_{\rho}(P) & =\mathrm{i} 2 M \int \frac{d^{4} q}{(2 \pi)^{4}}\left(D_{\mathcal{B}}^{\rho}(P-q) D_{\mathcal{M}}^{\rho}(q)-D_{\mathcal{B}}^{0}(P-q) D_{\mathcal{M}}^{0}(q)\right),
\end{aligned}
$$

where $D_{\mathcal{B}(\mathcal{M})}^{\rho}$ are the baryon (meson) propagator calculated at finite density $\rho$.

We can now solve the on-shell Bethe-Salpeter equation in nuclear matter for the in-medium amplitudes using the spin-flavor extended WT interaction that respects HQSS constraints:

$$
T^{\rho(C S I J)}=\frac{V^{C S I J}}{1-V^{C S I J} G_{\rho}^{C S I J}}
$$




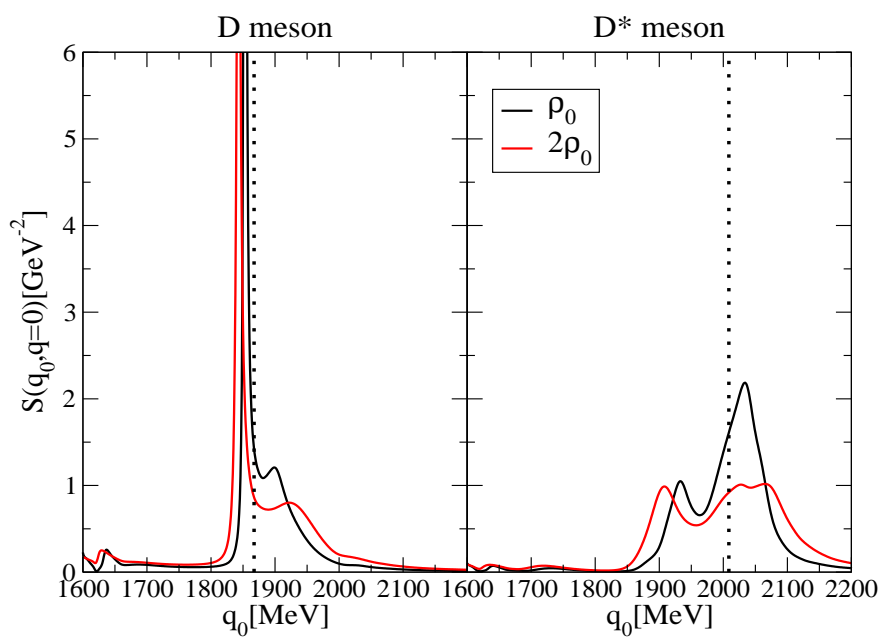

Fig. 4. The $D$ and $D^{*}$ spectral functions for $\rho_{0}$ and $2 \rho_{0}$ at $\vec{q}=0 \mathrm{MeV} / \mathrm{c}$. Taken from Ref 68 .

In particular, the self-energies of open charm $D$ and $\bar{D}$ and their HQSS partners $D^{*}$ and $\bar{D}^{*}$ can be calculated simultaneously in a self-consistent manner. These selfenergies are obtained by integrating the effective interaction in matter, $T_{D\left(D^{*}\right) N}^{\rho}$, over the nucleon Fermi sea,

$$
\begin{aligned}
\Pi_{D(\bar{D})}\left(q_{0}, \vec{q}\right)= & \int \frac{d^{3} p}{(2 \pi)^{3}} n(\vec{p})\left[T_{D(\bar{D}) N}^{\rho}{ }^{(I=0, J=1 / 2)}+3 T_{D(\bar{D}) N}^{\rho}{ }^{(I=1, J=1 / 2)}\right] \\
\Pi_{D^{*}\left(\bar{D}^{*}\right)}\left(q_{0}, \vec{q}\right)= & \int \frac{d^{3} p}{(2 \pi)^{3}} n(\vec{p})\left[\frac{1}{3} T_{D^{*}\left(\bar{D}^{*}\right) N}^{\rho}{ }^{(I=0, J=1 / 2)}+T_{D^{*}\left(\bar{D}^{*}\right) N}^{\rho}{ }^{(I=1, J=1 / 2)}+\right. \\
& \left.\frac{2}{3} T_{D^{*}\left(\bar{D}^{*}\right) N}^{\rho}{ }^{(I=0, J=3 / 2)}+2 T_{D^{*}\left(\bar{D}^{*}\right) N}^{\rho(I=1, J=3 / 2)}\right]
\end{aligned}
$$

where $P_{0}=q_{0}+E_{N}(\vec{p}, T)$ and $\vec{P}=\vec{q}+\vec{p}$ are the total energy and momentum of the meson-nucleon pair in the nuclear matter rest frame, and $\left(q_{0}, \vec{q}\right)$ and $\left(E_{N}, \vec{p}\right)$ stand for the energy and momentum of the meson and nucleon, respectively, in this frame. The self-energy is determined self-consistently since it is obtained from the in-medium amplitude which contains the meson-baryon loop function, and this quantity itself is a function of the self-energy. Then, the meson spectral function reads

$$
S_{M}\left(q_{0}, \vec{q}\right)=-\frac{1}{\pi} \frac{\operatorname{Im} \Pi_{M}\left(q_{0}, \vec{q}\right)}{\left|q_{0}^{2}-\vec{q}^{2}-m_{M}^{2}-\Pi_{M}\left(q_{0}, \vec{q}\right)\right|^{2}} .
$$

for $M=D(\bar{D}), \bar{D}\left(\bar{D}^{*}\right)$.

The $D$ and $D^{*}$ spectral functions for $\rho_{0}$ and $2 \rho_{0}\left(\rho_{0}=0.17 \mathrm{fm}^{-3}\right)$ at zero momentum are displayed in Fig. 4. Any dynamically-generated resonance which strongly 
couples to $D N$ and/or $D^{*} N$ channels will appear as a resonant-hole state in the corresponding spectral function for energies around the mass of the resonance once the nucleon mass is subtracted. The peak of the $D$ meson spectral function mixes strongly with $\Sigma_{c}(2823) N^{-1}$ and $\Sigma_{c}(2868) N^{-1}$ states, where we have denote $N^{-1}$ as hole. These $\Sigma_{c}$ resonances are predictions of our model with no experimental confirmation yet. Note that these resonances have higher masses than those described in Sec. 3. These states were seen in the wider energy range explored in Ref. ${ }^{29}$ but do not come from the most attractive $\mathrm{SU}(6) \times$ HQSS representations. The $\Lambda_{c}(2595) N^{-1}$ is clearly visible in the low-energy tail. With regard to the $D^{*}$ meson, the $D^{*}$ spectral function incorporates the $J=3 / 2$ resonances, and the peak of the spectral function fully mixes with the $\Sigma_{c}(2902) N^{-1}$ and $\Lambda_{c}(2941) N^{-1}$ states. The $\Sigma_{c}(2902)$ can be identified with the experimental $\Sigma_{c}(2800), \frac{43}{4}$ as seen in Ref. ${ }^{29}$ while $\Lambda_{c}(2941)$ has no experimental correspondence yet. Again these states are not reported in Sec. 3 as they do not come from the most attractive $\mathrm{SU}(6) \times \mathrm{HQSS}$ representations. For both $D$ and $D^{*}$ mesons, the $Y_{c}\left(=\Lambda_{c}^{(*)}, \Sigma_{c}^{(*)}\right) N^{-1}$ modes tend to smear out and the spectral functions broaden with increasing phase space, as seen before in the $t \rightarrow 0$ vector-meson exchange model of Ref ${ }^{[15}$ Compared to the previous results for the $D$ meson spectral function of Refs. ${ }^{8,14][15][67}$ we find that all of them generate the $\Lambda_{c}(2595) N^{-1}$ for energies around $1600 \mathrm{MeV}$, however with different strength. Moreover, while the works of Refs. ${ }^{8 \mid 14][15 \mid 67]}$ also generate the experimental $\Sigma_{c}(2800)$ resonance, though, in different positions, the model based on the extended WT to SF with HQSS constraints contains not only this resonance but also a richer spectrum of states.

The optical potential for $D$ and $\bar{D}$ meson is shown in Fig. [5] Defined as

$$
V_{D(\bar{D})}(\rho, E)=\frac{\Pi_{D(\bar{D})}\left(q^{0}=m_{D(\bar{D})}+E, \vec{q}=0, \rho\right)}{2 m_{D(\bar{D})}},
$$

where $E=q^{0}-m_{D(\bar{D})}$ is the $D$ or $\bar{D}$ energy excluding its mass, and $\Pi_{D(\bar{D})}$ the meson self-energy, we observe in both cases a strong energy dependence close to the open-charm meson mass. In the case of the $D$ meson, this is due to the mixing of the peak of the spectral function with the $\Sigma_{c}(2823) N^{-1}$ and $\Sigma_{c}(2868) N^{-1}$ states. For the $\bar{D}$ meson, the presence of a bound state at $2805 \mathrm{MeV}$, discussed in Sec. 3 and found in Ref $[30$ almost at $\bar{D} N$ threshold, makes the potential also strongly energy dependent. This is in contrast to the $t \rightarrow 0$ vector-meson exchange model of Ref., $\frac{15}{5}$ also displayed in Fig. 5 for $\rho_{0}$ with the SU(4) label.

\section{Charmed mesons in nuclei}

In this final section we aim at reviewing possible experimental signatures of the in-medium modified open-charm properties. One possible scenario is the so-called $D$ and $\bar{D}$-meson bound states in nuclei.

$D$ and $\bar{D}$-meson bound states in ${ }^{208} \mathrm{~Pb}$ were predicted in Ref ${ }^{54}$ relying upon an attractive $D$ and $\bar{D}$-meson potential in the nuclear medium. This potential was 

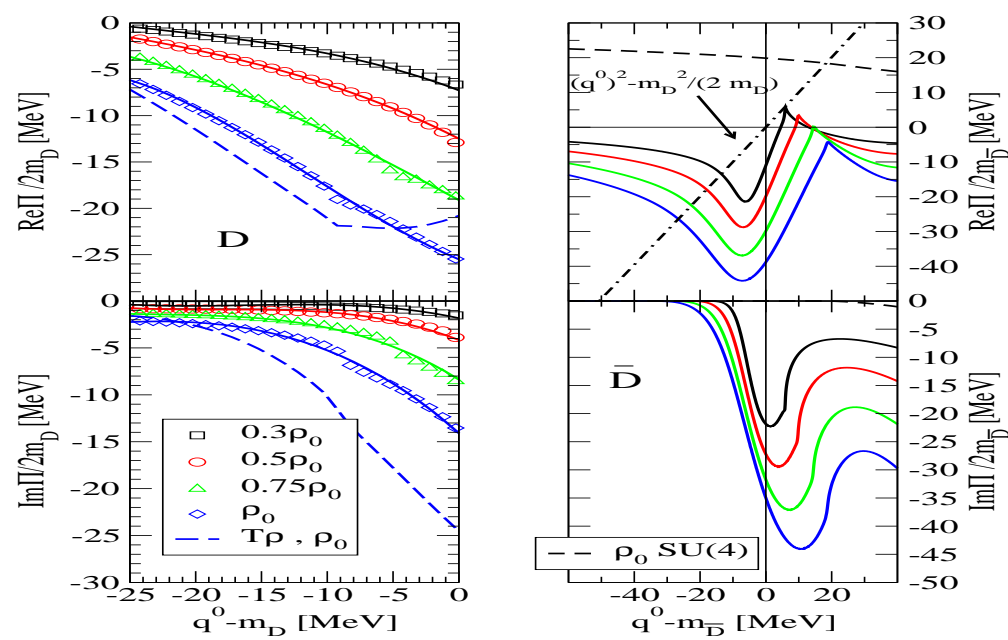

Fig. 5. The $D$ and $\bar{D}$ optical potential at $\vec{q}=0 \mathrm{MeV} / \mathrm{c}$ for different densities. Taken from Refs $[6970$

obtained within a QMC model ${ }^{55}$ The experimental observation of those bound states, though, might be problematic since, even if there are bound states, their widths could be very large compared to the separation of the levels. This is indeed the case for the potential derived from a SU(4) $t$-vector meson exchange model for $D$-mesons. 15,16

We look for $D$ - and $\bar{D}$ - nucleus bound states $\sqrt{69 \sqrt{70}}$ by solving the Schrödinger equation in the local density approximation:

$$
\left[-\frac{\nabla^{2}}{2 m_{\text {red }}}+V_{\text {coul }}(r)+V_{\mathrm{opt}}(r)\right] \Psi=(-B-i \Gamma / 2) \Psi .
$$

In this equation, $B$ is the binding energy $(B>0), \Gamma$ the width of the bound state and $m_{\text {red }}$ is the $D(\bar{D})$-nucleus reduced mass. $V_{\text {coul }}(r)$ is the Coulomb potential only for $D^{-}$mesons including the nucleus finite size and the Uehling vacuum polarization. We use the energy dependent optical potential $V_{\text {opt }}(r)=V((\rho(r), E)$, with $V(\rho, E)$ defined in Eq. (22) and shown in Fig. 5 . We apply the local density approximation in order to determine the relation between the density $\rho$ and the radius $r$ for each given nucleus density profile. Because the electromagnetic interaction is introduced by means of the minimal coupling prescription (to be consistent with gauge invariance and electric charge conservation), $V_{\text {coul }}(r)$ must be introduced wherever the energy is present. So the energy dependent optical potential is applied with argument $q^{0}=m-B-V_{\text {coul }}(r)$.

The question is whether $D$ and/or $\bar{D}$ will be bound in nuclei. We start by discussing $D$ mesons in nuclei. We observe that the $D^{0}$-nucleus states are weakly bound (see Fig. 6), in contrast to previous results using the QMC model ${ }^{[54}$ Moreover, those states have significant widths, $\frac{69}{\text { in }}$ particular for ${ }^{208} \mathrm{~Pb} .[54$ The best 


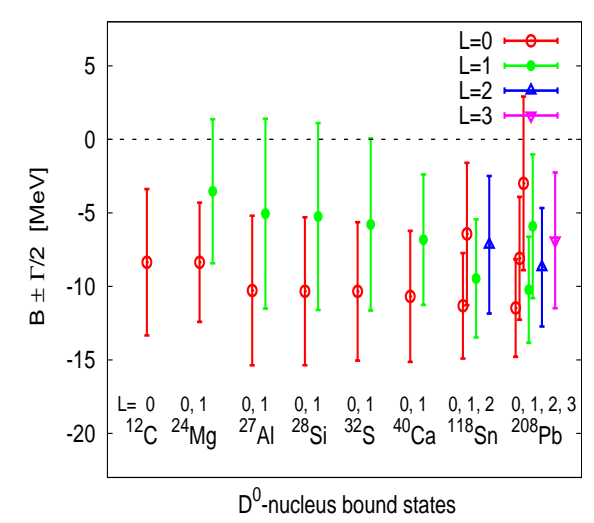

Fig. 6. $D^{0}$-nucleus bound states. Taken from Ref 69 .

chances for observation of bound states are in the region of ${ }^{24} \mathrm{Mg}$, provided an orbital angular momentum separation can be done, where there is only one $s$ - bound state and its half width is about a factor of two smaller than the binding energy. Only $D^{0}$-nucleus bound states are possible since the Coulomb interaction prevents the formation of observable bound states for $D^{+}$mesons.

Apropos of $\bar{D}$-mesic nuclei, not only $D^{-}$but also $\bar{D}^{0}$ bind in nuclei as seen in Fig. 7. The spectrum contains states of atomic and of nuclear types for all nuclei for $D^{-}$while, as expected, only nuclear states are present for $\bar{D}^{0}$ in nuclei. Compared to the pure Coulomb levels, the atomic states are less bound. The nuclear ones are more bound and may present a sizable width. ${ }^{70]}$ Moreover, nuclear states only exist for low angular momenta.

In Ref ${ }^{71}$ a bound state of $D N N$ is found with a large binding energy (bigger than $100 \mathrm{MeV}$ ) and a relatively small width. The existence of $D N$ (namely the $\left.\Lambda_{c}(2595)\right)$ and $D N N$ bound states, suggests that $D$-nucleus deeply bound states might also exist for larger nuclei. This would be consistent with the optical potential displayed in Fig. 3 of Ref., $\frac{68}{6}$ where a strongly attractive region is displayed around $200 \mathrm{MeV}$ below threshold. In the results reported in this review, however, only the region near threshold has been considered.

The information on bound states is very valuable to gain some knowledge on the charmed meson-nucleus interaction, which is of interest for PANDA at FAIR. The experimental detection of $D$ and $\bar{D}$-meson bound states is, though, a difficult task. For example, reactions with antiprotons on nuclei (Fig. 8) for obtaining $D^{0}$-nucleus states might have a very low production rate, as seen in Ref ${ }^{[69}$ Reactions but with proton beams, although difficult, seem more likely to trap a $D^{0}$ in nuclei $\frac{69}{6}$ 

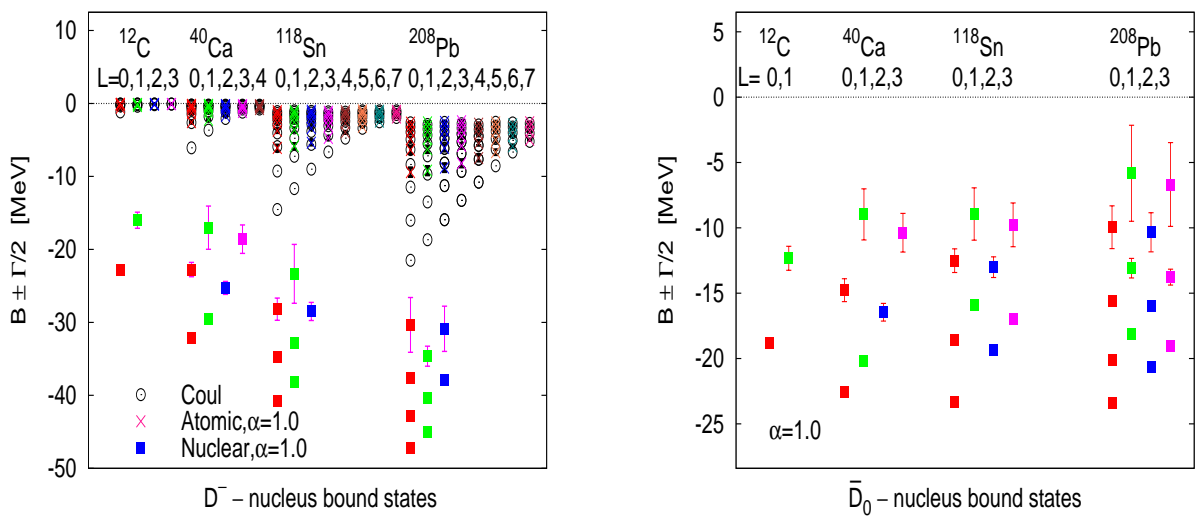

Fig. 7. $D^{-}$and $\bar{D}^{0}$ - nucleus bound states. Taken from Ref

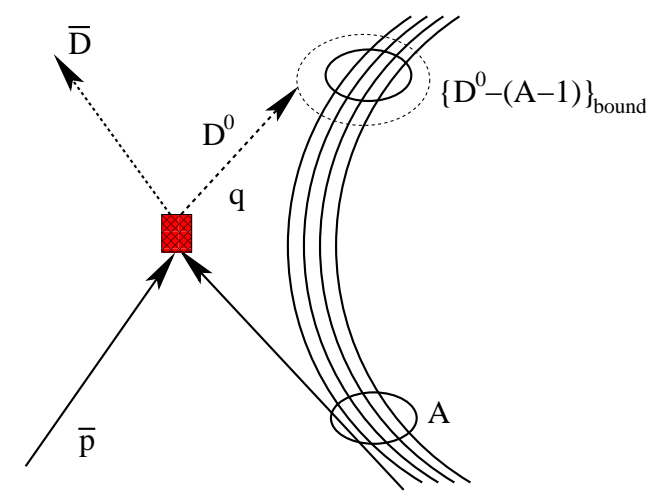

Fig. 8. Possible production of $D^{0}$-mesic nuclei with an antiproton beam. Taken from Ref 69 .

\section{Summary}

We have reviewed the properties of charmed mesons in dense matter and nuclei. We have started by discussing unitarized coupled-channel approaches in free space, paying a special attention to the model that takes, as bare interaction, effective Lagrangians that respect chiral symmetry in the light sector while HQSS in the heavy one. Within this scheme, several resonances have been generated dynamically by the $s$-wave interaction between pseudoscalar and vector meson multiplets with $1 / 2^{+}$and $3 / 2^{+}$baryons. Some of them have been identified with experimental results, such as the $\Lambda_{c}(2595)$, the $\Lambda_{c}(2625)$, the $\Xi_{c}(2790)$ or the $\Xi_{c}(2815)$ states, the last two forming a HQSS doublet. Moreover, we have overviewed the properties of open-charm mesons in nuclear matter by studying different self-consistent coupledchannel approaches and, in particular, the one which explicitely respects HQSS 
constraints. Finally, we have analyzed the experimental signatures of the in-medium properties of the open-charm mesons, such as the possible formation of charmed mesic nuclei in the context of FAIR.

\section{Acknowledgements}

The author warmly thanks C. Garcia-Recio, J. Nieves, O. Romanets and L.L. Salcedo for the careful reading of the manuscript. This research was supported by Ministerio de Ciencia e Innovación under contract FPA2010-16963, the EU HadronPhysics3 project (Grant Agreement No. 283286), the Ramon y Cajal Research Programme, and the FP7- PEOPLE-2011-CIG under Contract No. PCIG09-GA2011-291679.

\section{References}

1. http://home.web.cern.ch/about/accelerators/large-hadron-collider

2. http://www.gsi.de/ fair

3. T. Matsui and H. Satz, Phys. Lett. B 178 (1986) 416

4. http://www.lepp.cornell.edu/Research/EPP/CLEO/

5. http://belle.kek.jp/

6. http://www-public.slac.stanford.edu/babar/

7. E. Klempt and J. -M. Richard, Rev. Mod. Phys. 82 (2010) 1095

8. L. Tolos, J. Schaffner-Bielich and A. Mishra, Phys. Rev. C 70 (2004) 025203

9. L. Tolos, J. Schaffner-Bielich and H. Stoecker, Phys. Lett. B 635 (2006) 85

10. M. F. M. Lutz and E. E. Kolomeitsev, Nucl. Phys. A 730 (2004) 110

11. M. F. M. Lutz and E. E. Kolomeitsev, Nucl. Phys. A 755 (2005) 29

12. J. Hofmann and M. F. M. Lutz, Nucl. Phys. A 763 (2005) 90

13. J. Hofmann and M. F. M. Lutz, Nucl. Phys. A 776 (2006) 17

14. M. F. M. Lutz and C. L. Korpa, Phys. Lett. B 633 (2006) 43

15. T. Mizutani and A. Ramos, Phys. Rev. C 74 (2006) 065201

16. L. Tolos, A. Ramos and T. Mizutani, Phys. Rev. C 77 (2008) 015207

17. C. E. Jimenez-Tejero, A. Ramos and I. Vidana, Phys. Rev. C 80 (2009) 055206

18. J. Haidenbauer, G. Krein, U. G. Meissner and A. Sibirtsev, Eur. Phys. J. A 33 (2007) 107

19. J. Haidenbauer, G. Krein, U. G. Meissner and A. Sibirtsev, Eur. Phys. J. A 37 (2008) 55

20. J. Haidenbauer, G. Krein, U. G. Meissner and L. Tolos, Eur. Phys. J A 47 (2011) 18

21. J. -J. Wu, R. Molina, E. Oset and B. S. Zou, Phys. Rev. Lett. 105 (2010) 232001

22. J. -J. Wu, R. Molina, E. Oset and B. S. Zou, Phys. Rev. C 84 (2011) 015202

23. J. -J. Wu, T. -S. H. Lee and B. S. Zou, Phys. Rev. C 85 (2012) 044002

24. E. Oset, A. Ramos, E. J. Garzon, R. Molina, L. Tolos, C. W. Xiao, J. J. Wu and B. S. Zou, Int. J. Mod. Phys. E 21 (2012) 1230011

25. N. Isgur, M. B. Wise, Phys. Lett. B 232 (1989) 113

26. M. Neubert, Phys. Rep. 245 (1994) 259

27. A. V. Manohar and M. B. Wise, "Heavy Quark Physics", Camb. Monogr. Part. Phys. Nucl. Phys. Cosmol. 10 (2000) 1

28. J. -J. Wu and B. S. Zou, Phys. Lett. B 709 (2012) 70

29. C. Garcia-Recio, V. K. Magas, T. Mizutani, J. Nieves, A. Ramos, L. L. Salcedo and L. Tolos, Phys. Rev. D 79 (2009) 054004 
30. D. Gamermann, C. Garcia-Recio, J. Nieves, L. L. Salcedo and L. Tolos, Phys. Rev. D 81 (2010) 094016

31. O. Romanets, L. Tolos, C. Garcia-Recio, J. Nieves, L. L. Salcedo and R. G. E. Timmermans, Phys. Rev. D 85 (2012) 114032

32. C. Garcia-Recio, J. Nieves, O. Romanets, L. L. Salcedo and L. Tolos, Phys. Rev. D 87 (2013) 074034

33. C. Garcia-Recio, J. Nieves and L. L. Salcedo, Phys. Rev. D 74 (2006) 034025

34. C. Garcia-Recio, J. Nieves and L. L. Salcedo, Phys. Rev. D 74 (2006) 036004

35. H. Toki, C. Garcia-Recio and J. Nieves, Phys. Rev. D 77 (2008) 034001

36. C. Garcia-Recio, L. S. Geng, J. Nieves and L. L. Salcedo, Phys. Rev. D 83 (2011) 016007

37. D. Gamermann, C. Garcia-Recio, J. Nieves and L. L. Salcedo, Phys. Rev. D 84 (2011) 056017

38. C. Garcia-Recio, L. S. Geng, J. Nieves, L. L. Salcedo, E. Wang and J. -J. Xie, Phys. Rev. D 87 (2013) 096006

39. C. Garcia-Recio, J. Nieves, O. Romanets, L. L. Salcedo and L. Tolos, Phys. Rev. D 87 (2013) 034032

40. J. Nieves and E. Ruiz Arriola, Phys. Rev. D 64 (2001) 116008

41. C. Garcia-Recio, M. F. M. Lutz and J. Nieves, Phys. Lett. B 582 (2004) 49

42. T. Hyodo, D. Jido and A. Hosaka, Phys. Rev. C 78 (2008) 025203

43. J. Beringer et al. [Particle Data Group Collaboration], Phys. Rev. D 86 (2012) 010001

44. D. Jido, J. A. Oller, E. Oset, A. Ramos and U. G. Meissner, Nucl. Phys. A 725 (2003) 181

45. B. Wu and B. -Q. Ma, Phys. Rev. D 70 (2004) 034025

46. A. Aktas et al. [H1 Collaboration], Phys. Lett. B 588 (2004) 17

47. C. W. Xiao, J. Nieves and E. Oset, Phys. Rev. D 88 (2013) 056012

48. S. G. Yuan, K. W. Wei, J. He, H. S. Xu and B. S. Zou, Eur. Phys. J. A 48 (2012) 61

49. M. Gonin et al. [NA50 Collaboration], Nucl. Phys. A 610 (1996) 404C.

50. B. Zhang, C. M. Ko, B. -A. Li, Z. -w. Lin and B. -H. Sa, Phys. Rev. C 62 (2000) 054905

51. W. Cassing and E. L. Bratkovskaya, Nucl. Phys. A 623 (1997) 570

52. E. L. Bratkovskaya, W. Cassing and H. Stoecker, Phys. Rev. C 67 (2003) 054905

53. A. Sibirtsev, K. Tsushima, K. Saito and A. W. Thomas, Phys. Lett. B 484 (2000) 23

54. K. Tsushima, D. -H. Lu, A. W. Thomas, K. Saito and R. H. Landau, Phys. Rev. C 59 (1999) 2824

55. A. Sibirtsev, K. Tsushima and A. W. Thomas, Eur. Phys. J. A 6 (1999) 351

56. C. Fuchs, B. V. Martemyanov, A. Faessler and M. I. Krivoruchenko, Phys. Rev. C 73 (2006) 035204

57. R. Molina, D. Gamermann, E. Oset and L. Tolos, Eur. Phys. J. A 42 (2009) 31

58. P. A. M. Guichon, Phys. Lett. B 200 (1988) 235

59. A. Hayashigaki, Phys. Lett. B 487 (2000) 96

60. P. Morath, W. Weise and S. H. Lee, in Proceedings of the 17th Autumm school on QCD: Perturbative or Nonperturbative? Lisbon 1999, edited by L. S. Ferreira, P. Nogueira, and J. I. Silva-Marcos (World Scientific, Singapore, 2001), p. 425; W. Weise, in Proceedings of Hirschegg '01: Structure of Hadrons: 29th International Workshop on Gross Properties of Nuclei and Nuclear Excitations, Hirschegg, Austria, 14-20 Jan 2001, published in *Hirschegg 2001, Structure of hadrons*, p. 249

61. T. Hilger, R. Thomas and B. Kampfer, Phys. Rev. C 79 (2009) 025202

62. T. Hilger, B. Kampfer and S. Leupold, Phys. Rev. C 84 (2011) 045202

63. T. Hilger, T. Buchheim, B. Kampfer and S. Leupold, Prog. Part. Nucl. Phys. 
(2012) 188

64. A. Mishra, E. L. Bratkovskaya, J. Schaffner-Bielich, S. Schramm and H. Stoecker, Phys. Rev. C 69 (2004) 015202

65. A. Kumar and A. Mishra, Phys. Rev. C 81 (2010) 065204

66. A. Kumar and A. Mishra, Eur. Phys. J. A 47 (2011) 164

67. C. E. Jimenez-Tejero, A. Ramos, L. Tolos and I. Vidana, Phys. Rev. C 84 (2011) 015208

68. L. Tolos, C. Garcia-Recio and J. Nieves, Phys. Rev. C 80 (2009) 065202

69. C. Garcia-Recio, J. Nieves and L. Tolos, Phys. Lett. B 690 (2010) 369

70. C. Garcia-Recio, J. Nieves, L. L. Salcedo and L. Tolos, Phys. Rev. C 85 (2012) 025203

71. M. Bayar, C. W. Xiao, T. Hyodo, A. Dote, M. Oka and E. Oset, Phys. Rev. C 86 (2012) 044004 